\title{
Analysis and Solution of the Crack of the Outer Plate of the Door
}

\author{
Hua-Gang LIU ${ }^{1, a^{*}}$, Rui-Can HAO ${ }^{2, b}$ and Zhi-Xin FENG ${ }^{3, c}$ \\ Beijing Polytechnic, Beijing, China \\ aliuhuagang999@126.com, 'bhaoruican@163.com, ' zhixinfeng75@126.com \\ *Corresponding author
}

Keywords: Automobile panel, Door outer panel, Drawing die, Technology, Wrinkle, Crack.

\begin{abstract}
The stretching process is a complex plastic deformation process, the deformation zone is relatively large, metal flow, in the process of drawing prone to flange wrinkling and force transferring area of tensile deformation to scrap parts. Therefore, it is necessary to analyze the wrinkle, the root cause of the tension, in tool design and manufacture of stamping process should be attention, to improve drawing quality. The quality and the cost of the car body is very much, in the mold development stage is basically determined. This stage of product plan, process plan, mold preparation, etc., it has decided the cost of the mold, stamping parts material utilization, production efficiency, stamping parts quality, so in the body parts stamping die development stage for effective cost control and quality management is very necessary.
\end{abstract}

\section{Introduction}

The quality of automobile covering parts of the cost of high and low, mainly depends on the reasonable degree of stamping process and die structure, and the applicability of the punching die structure and economy, and in the design of punching die has been solved, when the door plate at the determination of mold structure, mainly on the basis of stamping process program, at the same time consider the feasibility of mould processing and repair the mold, the feasibility of the adjustment, the endurance of the tooling cost and stamping production security, etc. Stamping drawing when most avoid is material wrinkle, buckling can cause surface damage, greatly reduce the service life of the grinding tool[1].Found in the debugging process problems, analyze and solve problems, to accumulate experience, so as to improve the service life of the mold and improve product quality, the best products sent to the user.

\section{Car Door Plate Quality Requirements}

Car door plate (as shown in figure 1) is one of the typical automotive covering parts, have adornment sex commonly, for beautiful and easy, for example with a coherent decorated ridge, decorative rod, decoration pits, the surface is not allowed to have wrinkles, dent, ripping, scratches and other surface defects of destruction. 


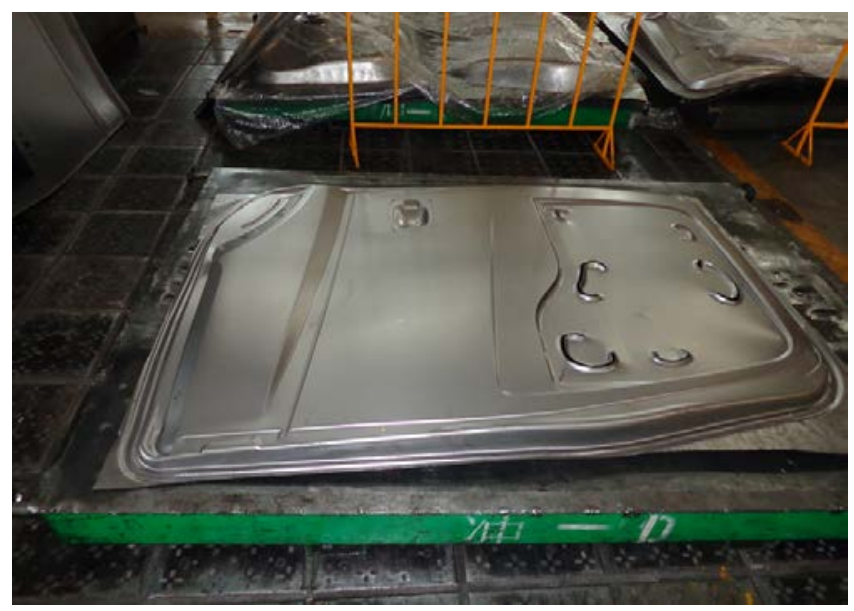

Figure 1 car door plate

Through the analysis of existing models of car door plate stamping parts liable to occur in the production of stamping the causes of defects and formulate corresponding measures, The trial production of the existing models, used in car door plate technology and die design.

Based on the car door plate forming process, at the same time considering the mould processing, repair, adjust the feasibility, determine the car door plate mould structure form, through the deep drawing die of car door plate, discusses the design of component technology and the complicated shape in the process of mould debugging are prone to cracking, wrinkling defects such as problem analysis, put forward to solve wrinkling, cracking defects measures [2].

\section{Car Door Plate Wrinkling Analysis of the Causes And Solutions}

(1) The wrinkling (figure 2) materials in the process of deep drawing him around the edge of the part, due to the large tangential stress, cause material loss of stability, make the product surface or edge cut to form the rough wrinkles, referred to as the wrinkling.
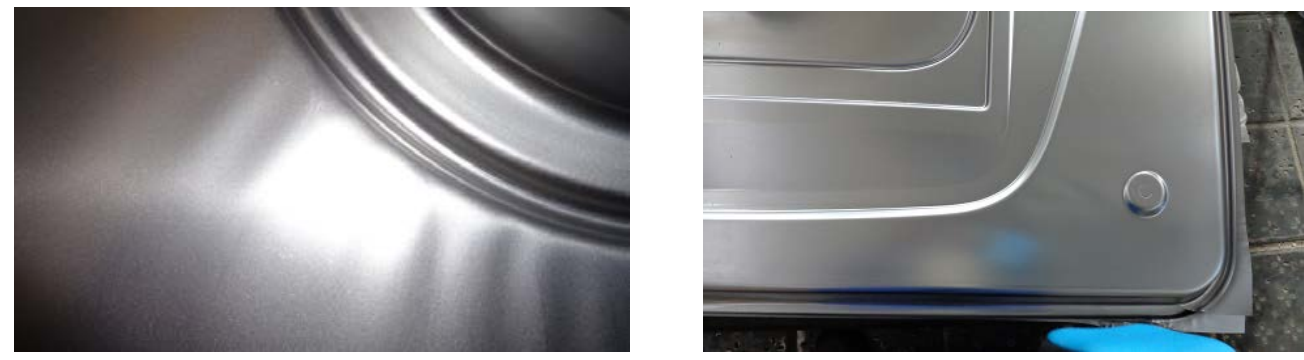

Figure 2 wrinkling defects

(2) The wrinkling is a common phenomenon in the stamping production and processing, in stamping production debugging should carefully analyse the causes of wrinkling, wrinkled, so as to work out concrete solutions to parts wrinkling.

(3) Through the car door plate analyzing the cause of wrinkling in the production, put forward own of car door plate, often appear wrinkles are analyzed.

(1) Convex cause wrinkles mainly because of the pressure force is too small.

(2) The convex and concave die fillet radius and the clearance is too large, too large radius decreased, the area of sheet metal in contact with the end of the punch and blank holder pressure material area of the circle, sheet metal dangling area increases, the wrinkle prone to instability.

(3) Top of flange wrinkling is the force that press a material single results, the cause of the force that press a material single pressure coil and the die is not parallel, bad material have burr, bad material surface is sundry. 
(4) The mold adjustment block height is not reasonable, causing the concave mold material surface and the blank holder gap uneven.

(5) The height of the hydraulic cushion plunger, cause material in deep drawing, the speed of flow is fast, slow.

(6) Lubricant use is not reasonable.

(4)Solve the car door plate wrinkling

(1) The appropriate increase pressure rim of material force.

(2) Refurbishment convex and concave die radius, increase the plate material and contact area of convex die and concave die face and blank holder ring of pressure area, reduce the phenomenon of instability, avoid wrinkling.

(3) To adjust the height of the blank holder ring plunger make pressure to coil and the concave mold material surface parallel.

(4) The height of the reasonable adjustment mold piece, keep the mold gap evenly.

(5) To replace hydraulic cushion plunger, the hydraulic cushion plunger highly consistent.

(6) The rational use of lubricant, smooth thin materials.

\section{Car Door Plate Cracking Analysis of the Causes And Solutions}

(1) The parts in the process of stamping tensile cracking (figure 3) is often encounter problems. There are many factors that can affect product cracking. The tensile properties of the material, the thickness of the material, drawing coefficient, concave and convex die radius, blank holder force and friction coefficient is part of the main causes of cracking [3].
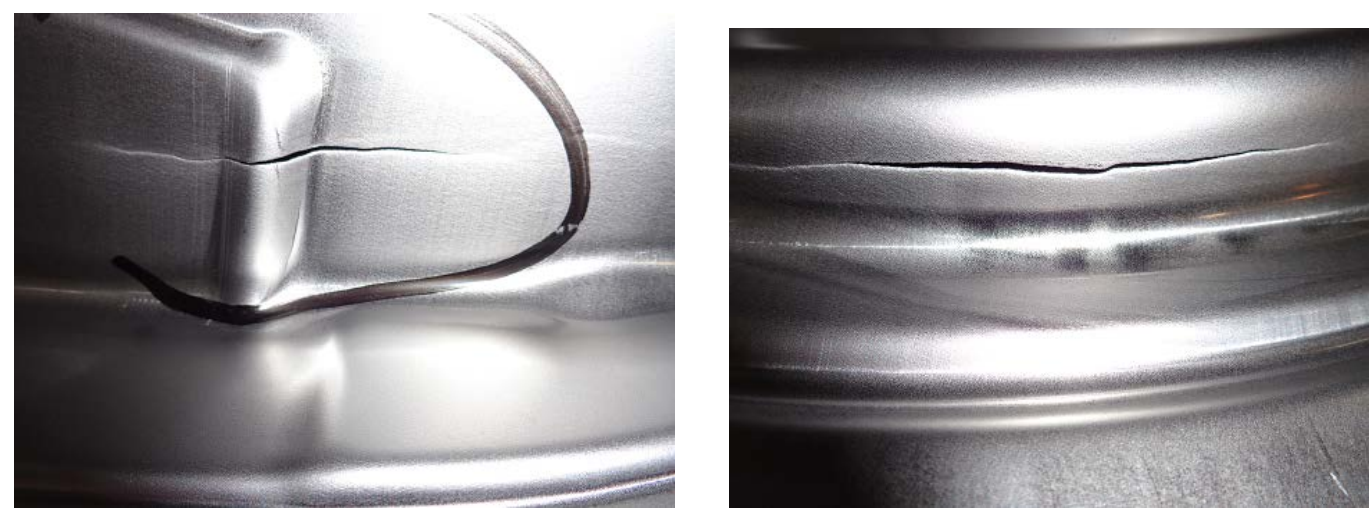

Figure.3 stamping tensile splitting tensile process

(2) The car door plate cracking caused by the analysis of the reason

(1) Material surface is not clean.

(2) Die working surface and the blank holder surface is not smooth.

(3) Pressure rim of the feed force is too small, the border region caused by pressure sheet wrinkling, sheet metal or hard can't through the draw bead, deep drawing sill, makes deep drawing part tensile stress increases sharply, also can cause cracking.

(4) Pressure rim of the feed force is too large, the border region caused by pressure plate or not through the draw bead, deep drawing, makes the deep drawing part tensile stress increases, leading to cracking.

(5) Concave mold material surface and uneven gap between blank holder ring.

(6) Pressure rim of the pressure force is too large.

(7) All landowners lubrication or inadequate lubrication of parts is not correct.

(3)The solving measures of car door plate cracking

(1) Clean the material surface or replacement

(2) The surface polishing die and blank holder ring work, make it smooth. 
(3) To adjust the height of block, make the concave die face and blank holder ring gap evenly.

(4) Adjust the pressure of the hydraulic cushion (cushion) make appropriate pressure rim of material force.

(5) The rational use of lubricating, and lubricant to clean, no impurity.

\section{Conclusion}

Stamping processing is the metal material in plastic deformation under the action of external force occur, obtain the required shape and size of the parts process, can make full use of the plasticity of metal and the minimum under the condition of deformation resistance, to obtain the required artifacts, formulation and implementation of the stamping process is the first important problem[4]. Understand material stamping performance, help to analysis of the production of quality problems related to the material performance. Need good equipment for automotive stamping parts and mold, good equipment and mould need better maintenance and maintenance, at the same time also should have a high level of mold adjuster. Mould adjuster in the actual production will find problems existing in production of stamping parts quality, put forward their own solutions, so as to produce high quality stamping parts.

\section{Reference}

[1]Qixiang Xue, Cold stamping practical technology: Beijing: mechanical industry press, 2006.1

[2]Xiang song qin, stamping production guide, Beijing: chemical industry press, 2008.8

[3]China made with stamping press association, The forging and stamping, paragraphs newsroom.2009.5

[4]Fuzhou Cai, Stamping mold design and manufacturing: Beijing: mechanical industry press, 2013.6 